\title{
Effect of Various Errors on the Spin Tune and Stable Spin Axis
}

\author{
W. W. MacKay
}

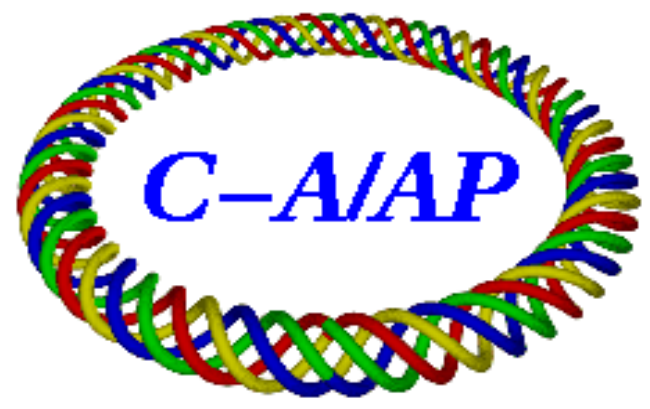

\section{Collider-Accelerator Department Brookhaven National Laboratory Upton, NY 11973}

Notice: This document has been authorized by employees of Brookhaven Science Associates, LLC under Contract No. DE-AC02-98CH10886 with the U.S. Department of Energy. The United States Government retains a nonexclusive, paid-up, irrevocable, world-wide license to publish or reproduce the published form of this document, or allow others to do so, for United States Government purposes. 


\title{
Effect of various errors on the spin tune and stable spin axis ${ }^{1}$
}

\author{
W. W. MacKay \\ Brookhaven National Laboratory, Upton, NY 11973, USA.
}

\begin{abstract}
Even though RHIC has two full Siberian snakes in each ring, there are various perturbations to the ideal case including orbit errors at the snakes, experiment solenoids, injection bumps, and interlaced horizontal-vertical bumps at the hydrogen jet polarimeter. These errors can cause depolarization by shifting the spin tune and tilting the stable spin direction away from vertical. Tilting of the stable spin axis can enhance horizontal depolarizing resonances. This paper presents preliminary results for some of these error scenarios, as well as their impact on the stable spin directions at STAR and PHENIX.
\end{abstract}

In order to vary the polarization direction for collisions, RHIC has four spin rotators around the STAR experiment and another four around the PHENIX experiment [1]. This arrangement allows independent control of the polarization axis for either beam and at either experiment. Each rotator consists of four helical dipoles with the inner and outer pairs each connected in series to separate power supplies [1]. In each ring, the rotators on either side of the experiment are powered with opposite currents to create a spin transparent insertion for each of the two IR's. Table 1 shows typical operating currents for longitudinal polarization at both experiments during the 2006 run [2] adjusted to minimize the transverse components of polarization with the local polarimeters at the experiments [3]. The predicted settings are from tracking through an analytic model of helical dipoles. The deviations in the settings for the Blue rotators around PHENIX are much larger than one would expect from magnet construction errors and measurement errors of transverse polarization components.

In order to understand discrepancies in the measured direction of the transverse polarization components for different running scenarios, I have resurrected code from an

TABLE 1. Typical rotator magnet currents for longitudinal polarization at $100 \mathrm{GeV}$ for STAR and PHENIX.

\begin{tabular}{lccccc}
\hline & \multicolumn{2}{c}{ PHENIX } & \multicolumn{2}{c}{ STAR } & \\
& Blue & Yellow & Blue & Yellow & Prediction \\
\hline$I_{\text {out }}[\mathrm{A}]$ & 214 & 221 & 211 & 215 & 221 \\
$I_{\text {in }}[\mathrm{A}]$ & 204 & 184 & 184 & 187 & 184 \\
\hline
\end{tabular}

${ }^{1}$ Work supported by Brookhaven Science Associates, LLC under Contract No. DE-AC02-98CH10886 with the U.S. Department of Energy. 
old online model called bl [4]. Spin tracking has been added in the new code blspin to explore the closed-orbit spin axis $\hat{n}_{0}$ and spin tune $v_{\mathrm{sp}}$ with various contributions including solenoids, bumps, orbit errors from rotators, snakes and quadrupole misalignments as well as dipole field errors. At present the program only deals with linear optics. The algorithms for spin motion were derived from the "standard theory" as presented in Ref. [5]. The approximations made for spin ignore the longitudinal field components of dipole fringes and the radial field components of solenoids. For RHIC at $100 \mathrm{GeV}$, these fringe field effects are small, although I plan to add these in the near future.

The twist angle of the helical dipoles as constructed for the snakes and rotators is systematically about $\Delta \alpha=0.4^{\circ}$ shy of a full $360^{\circ}$ rotation. For a single helical dipole with field $B_{0}$ and helical twist wavelength $\lambda$, the resulting vertical orbit deflection is approximately [6]

$$
\Delta y^{\prime} \approx-\frac{q B_{0}}{p} \frac{\lambda}{2 \pi} \sin \Delta \alpha,
$$

and is independent of the handedness of the helix. While being well within the design tolerances, for the rotators this does impart significant vertical deflections which must be locally corrected. Fig. 1 shows a simulated closed orbit with rotator steering errors corrected by local bumps in addition to closed separation bumps at four IR's.
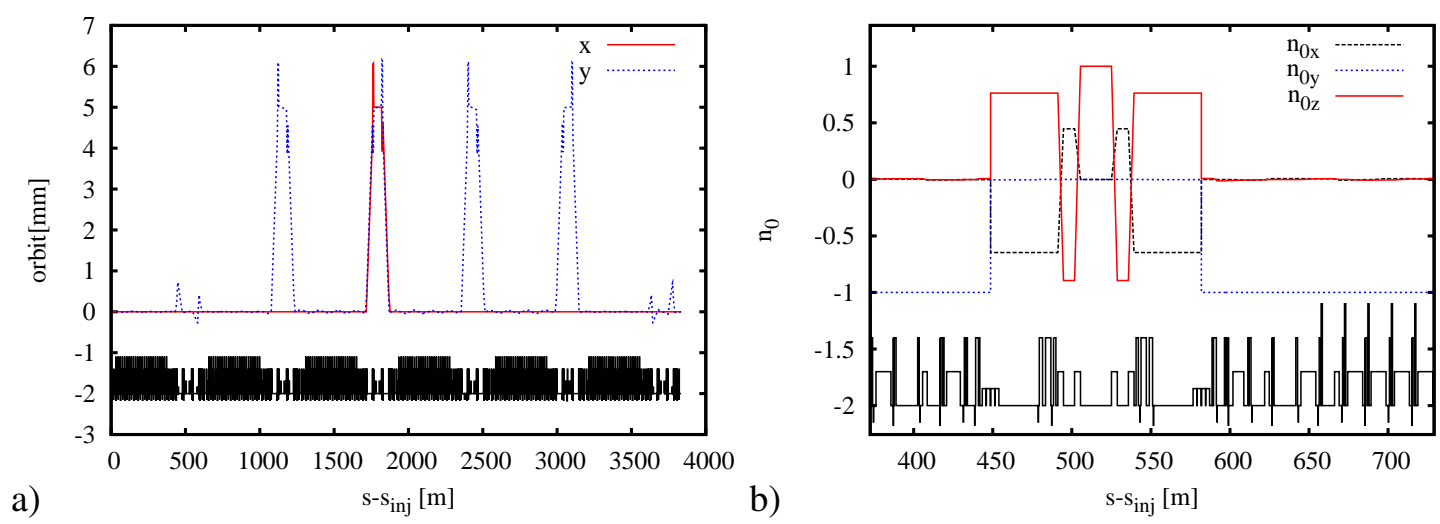

FIGURE 1. a) Closed orbit with vertical orbit errors from snakes and rotators and vertical separation bumps at four IR's. The errors from the rotators are corrected locally, but the smaller errors from the snakes remain uncompensated. The PHENIX and STAR interaction points are located at $515 \mathrm{~m}$ and $3710 \mathrm{~m}$, respectively. A horizontal bump at the hydrogen jet is also shown at $1793 \mathrm{~m}$. The snakes are located at 1034 and $2952 \mathrm{~m}$. b) Closed-orbit spin components through the PHENIX IR. The PHENIX rotators are shown centered about positions 449 and $582 \mathrm{~m}$, and the STAR rotators are similarly placed around the STAR IR. (Rotators and snakes are treated as thin elements in the model.)

Horizontal orbit errors through the snakes can cause tilts of the stable spin direction and spin-tune shifts [1] $\left|\Delta v_{\mathrm{sp}}\right|=\left|v_{\mathrm{sp}}-0.5\right| \approx 2 G \gamma\left|\Delta x^{\prime}\right| / \pi$, where $\Delta x^{\prime}=x_{2}^{\prime}-x_{1}^{\prime}$ is the deviation from parallel of the horizontal orbit at the snakes on opposite sides of the ring. For example, an error $\Delta x^{\prime}=0.1 \mathrm{mr}$ at the snakes would shift the spin tune by 0.012 . Orbit errors through the rotators can also cause sizable shifts. 
TABLE 2. Calculated spin tune and transverse components of $\vec{n}$ with various perturbations. The column headings are as follows. Rot: polarization direction at [PHENIX, STAR] - v for vertical and 1 for longitudinal; Sol for PHENIX and STAR solenoids - on or off; Vsep: vertical separation bumps at IR's 2, 4, 10, and 12 (not STAR and PHENIX); Hsep(Hjet): horizontal separation bump at IR 12; Misalign: random misalignment of arc quadrupoles; $v_{\mathrm{sp}}$ : spin tune; $n_{y}, n_{x}$ : vertical and radial components of $\hat{n}_{0}$ at CNI polarimeter. All calculations are for $100 \mathrm{GeV}$ with $\beta^{*}=1 \mathrm{~m}$ at STAR and PHENIX and $10 \mathrm{~m}$ at the other four IR's.

\begin{tabular}{rccccccrr}
\hline & Rot & Sol & Vsep & Hsep(Hjet) & Misalign & $v_{\mathrm{sp}}$ & $n_{y}$ & \multicolumn{1}{c}{$n_{x}$} \\
\hline 1 & $\mathrm{v}, \mathrm{V}$ & off & off & off & no & 0.500004 & 0.999999 & -0.000097 \\
2 & $\mathrm{v}, \mathrm{V}$ & on & off & off & no & 0.500011 & 0.999859 & 0.006818 \\
3 & $\mathrm{v}, \mathrm{V}$ & off & $5 \mathrm{~mm}$ & off & no & 0.500005 & 0.999795 & -0.000243 \\
4 & $\mathrm{v}, \mathrm{V}$ & on & $5 \mathrm{~mm}$ & off & no & 0.500012 & 0.999387 & 0.006673 \\
5 & $\mathrm{v}, \mathrm{V}$ & on & $5 \mathrm{~mm}$ & $5 \mathrm{~mm}$ & no & 0.499974 & 0.999398 & 0.002919 \\
6 & 1,1 & off & $5 \mathrm{~mm}$ & off & no & 0.499447 & 0.999816 & 0.001904 \\
7 & 1,1 & on & $5 \mathrm{~mm}$ & off & no & 0.496647 & 0.999817 & 0.001829 \\
8 & 1,1 & on & $5 \mathrm{~mm}$ & $5 \mathrm{~mm}$ & no & 0.496684 & 0.999817 & -0.001600 \\
9 & 1,1 & on & $5 \mathrm{~mm}$ & $5 \mathrm{~mm}$ & no & 0.496684 & 0.999817 & -0.001600 \\
10 & 1,1 & on & $5 \mathrm{~mm}$ & $5 \mathrm{~mm}$ & yes & 0.493656 & 0.999979 & -0.006179 \\
\hline
\end{tabular}

Table 2 shows calculated spin tune, and transverse spin components at the location of the $\mathrm{p}+\mathrm{C}$ CNI polarimeter (at $1864 \mathrm{~m}$ ) for various combinations of bumps, solenoids, rotator settings, and quadrupole misalignment. The first row shows a slight tune shift caused by the small vertical errors and path-length increase from the snakes. (The dispersion function is different at the two snakes.) The effect on spin tune from the experiment solenoids is negligible unless the rotators are turned on; compare rows 1-4 with 6 and 7. Vertical separation at IR's without rotators does not affect the spin, since the four horizontal bends for each IR crossing (two D0 and two DX magnets) have no quadrupoles between them. The last line of Table 2 shows an example of random misalignments of the arc quadrupoles (from Q8 to Q8). The random misalignments were generated with a flat distribution between $\pm 0.3 \mathrm{~mm}$ in both planes. This particular set of misalignments resulted in the rms orbit deviations (Fig. 2a) in the arcs of $\sigma_{x}=0.57 \mathrm{~mm}$ and $\sigma_{y}=0.42 \mathrm{~mm}$. The transverse components of $\hat{n}$ for this last case were $\left(n_{x}, n_{y}\right)=$ $(-0.0823,0.0023)$ for PHENIX and $(-0.0404,-0.0104)$ for STAR. For the last row, $\vec{n}_{0}$ was tilted away from the longitudinal by about $5^{\circ}$ at PHENIX.

At $100 \mathrm{GeV}$ the combined horizontal and vertical separation bumps with respective amplitudes $x$ and $y$ at IR 12 with the Hjet polarimeter do not make a significant shift of $v_{\mathrm{sp}}$, but at $250 \mathrm{GeV}$ (see Fig. 2b) the effect, proportional to $x y^{2}$, is larger.

Basically any spin rotation not about the vertical direction can, in principle, cause a distortion of spin tune and direction. Since rotations are noncommutative, the effects can add nonlinearly as demonstrated by combinations of rotators and solenoids and by combined horizontal and vertical separation bumps. Vertical orbit errors around the ring can cause tilts and spin-tune shift, and if the dice roll badly, then combined horizontalvertical orbit errors can compound this. For other random quadrupole misalignments with $\sigma_{x, y} \sim 0.5 \mathrm{~mm}$, in the arcs, I have calculated $\Delta v_{\mathrm{sp}}>0.01$.

Preliminary results using the new spin tracking code blspin show tilts similar to what has been reported at times by the $\mathrm{p}+\mathrm{C}$ CNI, STAR, and PHENIX polarimeters. 

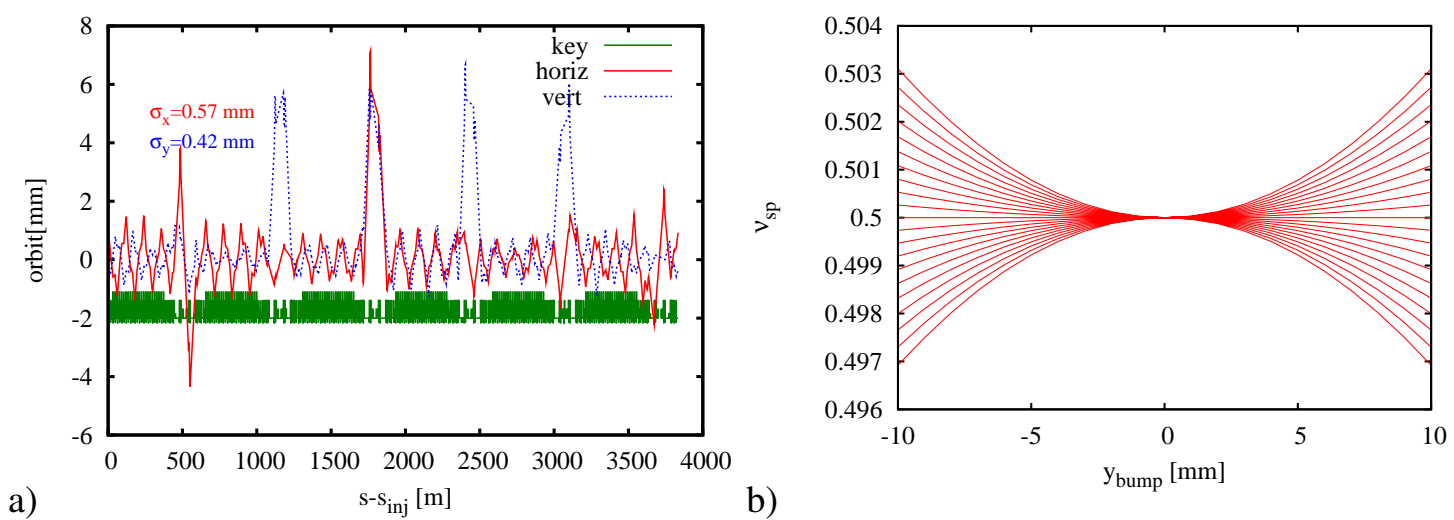

FIGURE 2. a) Closed orbit for conditions of last line of Table 2 (at $100 \mathrm{GeV}$ ). Notice the separation bumps at the 10, 12 (Hjet), 2, and 4 o'clock IR bumps. b) Effect on spin tune caused by combined horizontal and vertical bumps at the Hjet as calculated for $250 \mathrm{GeV}$ with ideal snakes and no rotators or errors. The curves are plotted for $1 \mathrm{~mm}$ steps in the horizontal bump from -10 to $10 \mathrm{~mm}$.

Since the original bl was written as an online code to simulate orbit steering, it can quickly simulate different alignment and bump configurations.

The setting of the Blue rotators at PHENIX relative to the predicted values corresponds to a vertical deflection of about $7-10^{\circ}$ away from the longitudinal. The simulated tilt at PHENIX for conditions in Fig. 2a was a horizontal deflection; however, so far I have not tried to simulate the actual orbit errors at snakes and rotators. By examining actual orbit data taken during longitudinal pp running, and trying to match the orbit slopes measured at the snakes and rotators, it may be possible to explain this discrepancy.

Future improvements to blspin will add contributions to spin precession from the longitudinal fringe contributions of bend magnets and the radial fringe fields of the solenoids. Higher order multipoles will be added for both orbit and spin calculations, in particular sextupoles. Calculation of the invariant spin field $\hat{n}$ for phase space points other than the closed orbit will be explored. I eventually plan to develop a version with spin-1 spinors for deuterons.

I would like to thank D. Barber, A. U. Luccio, S. Mane, C. Montag, V. Ptitsyn, and T. Roser for fruitful discussions. During the workshop, Des Barber and others pointed out the need for inclusion of spin precession from the longitudinal component of the dipole end fields in dealing with low energy deuterons.

\section{REFERENCES}

1. I. Alekseev et al., Configuration Manual Polarized Proton Collider at RHIC, BNL, (2006). URL:http: / / www . rhichome.bnl.gov/RHIC/Spin/design/CMan/CMan.pdf

2. V. Ptitsyn et al., "RHIC Performance as Polarized Proton Collider in Run-6", PAC06 (2006).

3. W. W. MacKay et al., "Commissioning Spin Rotators in RHIC", PAC03, 1697 (2003).

4. W. W. MacKay, “An Interactive Beam Line Simulator Module for RHIC”, PAC97, 2541 (1997).

5. S. R. Mane, Yu. M. Shatunov, and K. Yokoya, Rep. Prog. Phys., 68, 1997 (2005).

6. M. J. Syphers, "Field Quality Issues for RHIC Helical Dipole Magnets", (1995). URL: http://www.rhichome.bnl.gov/RHIC/Spin/spinnotes/sn015.pdf 